\title{
Ripensare il nesso tra apprendimento all'università e apprendi- mento al lavoro per favorire l'occupazione giovanile. Una ri- cerca sulla formazione di mentori competenti nei percorsi di ap- prendistato \\ Bridging the gap between academic learning and workplace \\ learning to foster youth employment. A research project on supporting the competent mentoring in apprenticeship Roberta Piazza*, Simona Rizzari ${ }^{* *}$
}

\begin{abstract}
Riassunto
Nel mondo digitale contemporaneo lo skills mismatch e la mancanza di opportunità di lavoro per i giovani rappresentano sfide non agevoli da affrontare. Per far fronte a tali difficoltà si ritiene che l'apprendistato possa giocare un ruolo chiave, in grado di consentire ai giovani di acquisire le competenze necessarie a facilitare la loro transizione dall'educazione al lavoro. La pedagogia è pertanto chiamata a riflettere sulla possibilità di pensare a modelli di apprendimento integrati, in grado di stabilire connessioni tra istruzione/formazione e lavoro con modalità nuove.

Tra i criteri per apprendistati efficaci e di qualità appare centrale il supporto pedagogico, offerto da personale interno alle imprese, con l'incarico di collaborare strettamente con le istituzioni formali. Sebbene l'importanza del mentoring nell'apprendistato sia ampiamente riconosciuta, la ricerca non ha sempre prestato adeguata attenzione alle competenze educative che i mentori dovrebbero possedere per preparare gli studenti quali learning agent. Lo studio qui presentato intende fornire un contributo di ricerca, definendo un modello di formazione per mentori. Il saggio - che descrive il progetto di ricerca internazionale ApprEnt - presenta il prototipo elaborato per la formazione di mentori competenti.

Parole chiave: disoccupazione, giovani, apprendimento formale, apprendimento al lavoro, apprendistato, mentoring.
\end{abstract}

Articolo sottomesso: 30/03/2020, accettato: 16/05/2020

\footnotetext{
* Professore ordinario di Pedagogia generale e sociale presso l'Università degli Studi di Catania.E-mail: r.piazza@unict.it.

** Assegnista di ricerca presso l'Università degli Studi di Catania. E-mail: simona.rizzari@unict.it.

I paragrafi 1, 2 e 4 sono di Roberta Piazza. I paragrafi 3, 5 e 6 sono di Simona Rizzari.
}

Doi: $10.3280 /$ ess2-2020oa9480 


\begin{abstract}
In today's digital world skills mismatch and lack of employment opportunities for young people around the world constitute serious challenges. In response to these challenges, there is increasing recognition of the key role that apprenticeships can play in enabling young people to acquire the relevant competencies to facilitate their transition from the world of education to the world of work.

Pedagogy is therefore called to reflect on the possibility of thinking about integrated learning models that are based on the possibility of establishing connections between education/training and employment in new ways.

Among the criteria for effective and quality apprenticeships, pedagogical support, offered by staff within companies, with the task of working closely with formal institutions, appears central. Although the importance of mentoring in apprenticeship is well established, research has not always paid adequate attention to the pedagogical skills that mentors should possess to prepare students as agentic learners. Through our study we contribute to the body of research by defining a training model for mentors involved in the apprenticeship scheme. The study - describing an international research project (ApprEnt) includes findings about pedagogic practices for training of competent mentors.
\end{abstract}

Keywords: unemployment, young people, formal learning, learning at work, apprenticeship, mentoring.

\title{
1. Giovani alla ricerca del lavoro tra skill mismatch e disoccupazione
}

Le due principali sfide per l'istruzione e la formazione in Europa sono rappresentate dall'elevata disoccupazione tra i giovani e dalla carenza di competenze di livello superiore richieste dal mercato di lavoro.

Il tasso globale di disoccupazione giovanile è quasi tre volte superiore al tasso di disoccupazione degli adulti (ILO, 2018). Nell'Unione europea, nel 2018, più di 5.5 milioni di giovani (15-24 anni) non erano né occupati né inseriti in percorsi formativi (NEET); nel 2019 si sono registrati più di 3.3 milioni di disoccupati nella medesima fascia di età ${ }^{1}$.

L'ingresso nel mondo del lavoro per i giovani - considerati una popolazione a rischio al momento del passaggio dalla scuola al lavoro - appare segnato da

${ }^{1} \mathrm{Cfr}$. https://ec.europa.eu/social/main.jsp?catId=1036 (ultima consultazione 22/03/2020). Sebbene il tasso di disoccupazione giovanile sia diminuito - dal 24\% nel 2013 a meno del $15 \%$ nel 2019 - nell'UE è ancora molto elevato (con punte di oltre il 30\% in molti paesi) e più del doppio del tasso di disoccupazione complessivo (meno del 7\%), con profonde differenze tra i diversi paesi. 
molteplici difficoltà. Sono esposti a un rischio più elevato di disoccupazione rispetto ai lavoratori più anziani; hanno maggiori probabilità di passare ricorsivamente dalla disoccupazione alla formazione, al lavoro; accedono più facilmente a occupazioni temporanee o precarie (Quintini et al., 2007). Forme nuove ed emergenti di lavori "atipici" dominano le prime esperienze del mercato del lavoro dei giovani (ILO, 2016). La loro limitata conoscenza del mercato del lavoro - spesso accompagnata dalla mancanza di servizi di collocamento in grado di fornire un accompagnamento su misura ai giovani, in particolare per i più vulnerabili - aumenta il rischio di lavori non qualificati o caratterizzati da condizioni di lavoro inadeguate (Frazer and Marlier, 2010). In effetti, nel periodo di transizione dalla scuola al lavoro, i giovani tendono a conoscere le proprie capacità e preferenze attraverso lo job shopping (Johnson, 1978; Topel and Ward, 1992), con conseguenti tassi più elevati di turn-over e periodi più frequenti di disoccupazione. Al medesimo tempo, le imprese di solito affrontano maggiori costi di investimento e minori costi di licenziamento quando assumono giovani lavoratori, rendendo la situazione del mercato del lavoro giovanile più sensibile alle fluttuazioni dal punto di vista della domanda (Caliendo and Schmidl, 2016).

Una quota crescente di lavori richiede competenze complesse e padronanza delle nuove tecnologie, come risultato dei cosiddetti "mega trends": innovazione tecnologica, cambiamenti nella divisione globale del lavoro, nell'organizzazione delle aziende, cambiamenti demografici e nei modelli di consumo (OECD, 2017a). Robotica, automazione, intelligenza artificiale, machine learning, l'avvento dei Big Data stanno determinando profondi cambiamenti nel mercato del lavoro (Frey and Osborne, 2013; CEDEFOP, 2016a; OECD, 2017b). La ricerca di skill richieste nella quarta rivoluzione industriale pone nuove sfide a un sistema già in difficoltà nell'identificare il lavoro adeguato alle competenze possedute. Il World Economic Forum ci informa che entro il 2022 le competenze chiave richieste per svolgere la maggior parte dei lavori cambieranno, in media, del 42\% (WEF, 2019, p. 1) ${ }^{2}$. Tutto ciò, mentre il concetto stesso di percorso lineare di carriera, basato su "impara, lavora, vai in pensione", è ormai superato (Purcell et al., 2013).

A fare le spese dell'attuale sistema di lavoro sono spesso le fasce giovanili più deboli, che assistono, impotenti, al rafforzamento delle disuguaglianze socio-economiche. Il successo nell'istruzione primaria, secondaria e terziaria rimane uno dei principali fattori predittivi dell'inclusione socioeconomica a lungo termine (OECD, 2017a; Dietrichson et al., 2017), sebbene non sia solo

\footnotetext{
${ }^{2}$ In Italia è previsto che il $15,2 \%$ dei lavori è a elevato rischio di automatizzazione, e un ulteriore $35,5 \%$ potrà sperimentare cambiamenti significativi in conseguenza dell'introduzione delle nuove tecnologie (OECD, 2019, p. 13).
} 
la quantità del percorso educativo, ma la qualità del sistema educativo che richiede di essere presa in considerazione nel valutare le competenze acquisite (Psacharopoulos and Patrinos, 2018).

Certamente, possedere forza lavoro qualificata è un prerequisito per la produttività e l'occupabilità e costituisce una pietra miliare per l'innovazione e la competitività delle imprese e degli stati. Pertanto, l'attenzione all'istruzione e gli investimenti per l'acquisizione delle competenze funzionali al lavoro sono stati posti alla base della strategia di molti paesi per superare la crisi e dare impulso alla crescita (Commissione europea, 2013a). Se tuttavia l'attenzione allo sviluppo delle skill, relativa ai diversi livelli di istruzione, è costantemente presente a livello politico nei paesi europei, le differenze tra le nazioni sono spesso considerevoli, in particolare per ciò che riguarda le competenze associate al titolo di studio: le economie dell'Europa meridionale, ad esempio, tra cui Portogallo, Spagna, Italia e Grecia, hanno tutte un terzo o più delle loro popolazioni in età lavorativa con titoli inferiori a quelli dell'istruzione secondaria superiore (Zanazzi, 2018).

L'Italia, inoltre, non solo è in coda tra i Paesi avanzati per percentuale di laureati, ma ha anche uno dei più allarmanti livelli mondiali di "disallineamento" tra i percorsi di studio scelti dai giovani e le esigenze del mercato del lavoro. Se confrontata con la Germania, come ci mostrano i dati di un recente studio di IGIER - Bocconi (Giustinelli, Pavoni and Anelli, 2019, p. 30) «l'Italia ha solo la metà dell'offerta di laureati in economia e ingegneria e più del doppio dell'offerta di laureati in arti, discipline umanistiche e scienze sociali. Particolarmente negativo è il confronto tra l'offerta di laureati nelle ITC, un settore che sappiamo fondamentale nello sviluppo economico recente e futuro»».

La centralità dell'automazione ha in effetti condotto all'accelerazione della domanda di lavoratori qualificati (skill biased technical change) (CEDEFOP, 2018) e alla conseguente progressiva riduzione della domanda di compiti cognitivi e manuali routinari (Brunello and Wruuck, 2019). Mai come prima l'educazione e la formazione si trovano impegnate nel compito di sostenere $\mathrm{i}$ giovani nell'affrontare le sfide del mercato del lavoro, orientandoli verso scelte di studio e di carriera più consapevoli. Fondamentale il coinvolgimento e la collaborazione di tutti gli attori coinvolti (famiglie, istituzioni formali, mondo del lavoro) nell'acquisire e interpretare le informazioni alla scelta, così da ridurre o alleviare lo skills mismatch (Giustinelli, Pavoni and Anelli, 2019, p. 19).

\section{L'integrazione sistemica di formazione e lavoro: il contributo della pedagogia}

Se la ricerca ha evidenziato che il ruolo giocato dai servizi di guidance è 
fondamentale nello skill matching e l'orientamento rappresenta una delle politiche di maggiore successo per l'occupazione (de Koning and Peers, 2007; CEDEFOP, 2015, 2016c), un preciso impegno della pedagogia è dato dal ripensamento dei tradizionali sistemi di formazione e dal rafforzamento di modelli capaci di integrare educazione formale e apprendimento basato sul lavoro.

Appare evidente che il possesso di competenze non adeguate non può essere risolto esclusivamente rafforzando o riformando la formazione professionale $\mathrm{o}$ fornendo alle persone competenze specifiche in funzione del lavoro. All'interno dei percorsi di carriera, in effetti, tutte le abilità e le competenze acquisite - a causa dei cambiamenti strutturali e organizzativi nel mercato del lavoro dovranno essere aggiornate e riviste più volte (Brunello and Rocco, 2017), pena la loro obsolescenza. Se in Europa i sistemi di formazione professionale sono stati considerati per lungo tempo maggiormente capaci di favorire un'agevole transizione dei giovani al lavoro rispetto ai percorsi educativi scolastici o accademici (Levels et al., 2014), esistono evidenze empiriche che dimostrano come i percorsi educativi formali, basati sull'acquisizione di conoscenze generali e competenze di base, integrati a esperienze di lavoro, siano più vantaggiosi in termini di riduzione dell'overskilling nel corso della carriera (Verhaest and Omey, 2013; Verhaest et al., 2018). La combinazione tra formazione generale e apprendimento sul posto di lavoro sembra produrre migliori effetti in termini di acquisizione di competenze. Nel tempo, le competenze derivanti da una formazione generale possono essere più vantaggiose, poiché sono quelle che consentono ai lavoratori di adattarsi alle continue richieste di modifica e aggiornamento delle loro skill. Le esperienze in contesti lavorativi concorrono a far acquisire competenze trasversali fondamentali per l'esercizio della pratica professionale. Per quanto l'istruzione formale non sembra ancora ben attrezzata a fornire esperienze di apprendimento sul luogo di lavoro (Cappelli, 2014; Brunello and Wruuck, 2019), modelli di apprendimento capaci di combinare percorsi educativi formali con l'esperienza nei contesti lavorativi sembrano rappresentare una risposta positiva all'inserimento lavorativo, per quanto ancora non semplici da attuare in EU (Wieland, 2018).

La pedagogia è pertanto chiamata a riflettere sulla necessità di pensare a modelli di apprendimento che si basino sulla possibilità di stabilire connessioni tra istruzione/formazione e occupazione in modalità nuove, al fine di creare ecosistemi e modelli integrati di apprendimento tra istruzione e lavoro. La consapevolezza acquisita è che le competenze fondamentali per affrontare le sfide del lavoro e della vita quotidiana provengono solo in parte dall'educazione formale (Federighi, 2012, p. 18). È soprattutto l'apprendimento nei luoghi lavoro, in modelli integrati con i contesti formali e a forte carattere riflessivo, a sostenere i giovani nel «finding ways to do better while doing the work» (Edmonton and Saxberg, 2017, p. 2). 
Pertanto, per consentire ai giovani di apprendere, disapprendere e riapprendere le competenze necessarie a inserirsi e a rimanere nel mercato del lavoro (Piazza, 2019), è opinione condivisa che i modelli di apprendimento fondati sul lavoro possano contribuire a formare lavoratori più consapevoli della loro learning agency (Ericsson, 2006; Lave and Wenger, 2006; Tomassini, 2016) e, quindi, maggiormente disponibili al cambiamento. Alla pedagogia il compito di sperimentare progettualità educative che siano capaci di integrare apprendimento e lavoro, fuori dai contesti formali tradizionali, e che consentano ai lavoratori di acquisire e utilizzare strumenti concettuali capaci di favorire una costante azione riflessiva (Schön, 1993; Moon, 2013) sull'apprendimento messo in atto e sulle esperienze realizzate.

Non che la pedagogia sia estranea alla riflessione sul lavoro. Autori e prospettive pedagogiche hanno concentrato da tempo l'attenzione sul tema del lavoro e della sua dimensione intrinsecamente educativa e formativa: gli scritti di Pestalozzi (1801), di Kerschensteiner $(1912,1926)$, di Dewey (1916), fra i molti, - da considerare fra le fonti teoriche di una pedagogia del lavoro -, offrono importanti elementi di riflessione pedagogica. Se la pedagogia scientifica, definitasi a partire dall'Ottocento, ha dedicato maggiore attenzione alla scuola rispetto ai temi del lavoro e della formazione al lavoro (Moscato, 2016), e il dualismo tra formazione classica e formazione tecnica e professionale ha acceso il dibattito fino alle epoche più recenti (Franchi, 1979), nel corso degli ultimi decenni la pedagogia ha ricontestualizzato la sua attenzione al lavoro (Bocca, 1998; Bertagna, 2006, 2011; Costa, 2011; Alessandrini, 2014), collocandola all'interno della società conoscitiva.

Oggi occuparsi di lavoro e di apprendimento significa per la pedagogia rielaborare i contributi delle scienze che da sempre si occupano di lavoro (sociologia, economia, diritto, ingegneria...) (Boffo, 2012), riconsiderare i mutati significati connessi al lavoro e alle sue trasformazioni, dialogare con i nuovi scenari delle tecnologie integrate al lavoro. L'orizzonte di senso è dato dalla necessità di orientare la riflessione pedagogica sempre più verso l'elaborazione di un pensiero critico-riflessivo sui temi legati al cambiamento nel mondo del lavoro e sul collegamento fra formazione generale e apprendimento work-based. I contesti lavorativi, investiti da processi formativi intenzionali, in costante dialogo con i sistemi formali, possono così diventare luoghi di trasformazioni personali e collettive e setting di elaborazione di azioni consapevoli volte alla costruzione delle scelte professionali e di vita.

\section{Costruire ponti tra università e lavoro: il work-based learning}

I profondi cambiamenti intervenuti nell'organizzazione del sistema lavora- 
tivo hanno determinato la progressiva ridefinizione delle finalità e degli obiettivi del sistema universitario, ponendolo dinanzi a una serie di interrogativi e di sfide inerenti in particolare all'ampliamento dell' accesso, alla strutturazione del curriculum, ma soprattutto alle competenze in uscita (learning outcomes) che gli studenti dovrebbero possedere ai fini della spendibilità sul mercato del lavoro del titolo conseguito (Brennan, King and Lebau, 2004). Per le imprese, d'altra parte, come rileva opportunamente Federighi (2012), la richiesta crescente di produzione di conoscenza al proprio interno, finalizzata allo sviluppo e all'innovazione, ha sollecitato la ricerca di efficaci modalità di gestione dei processi formativi incorporati nel lavoro (embedded learning). Tale approccio ha sfatato l'idea che la formazione in impresa si identifichi semplicemente «con l'insieme di processi che si sviluppano per il solo fatto di essere immersi in un ambiente» (p. 21), evidenziando come essa richieda, piuttosto, la ri-concettualizzazione delle esperienze lavorative alla luce delle conoscenze teoriche possedute.

Se è vero, come si è detto, che la combinazione fra formazione terziaria e apprendimento basato sul lavoro rappresenta una possibile soluzione, - poiché favorisce l'accesso e la partecipazione all'istruzione superiore anche alle categorie di studenti più svantaggiati e agli studenti lavoratori, estendendo l'acquisizione di competenze di livello superiore a un numero più elevato di individui e migliorandone l'occupabilità -, è pur vero che la costituzione di proficui partenariati tra università e imprese non è un compito facile. Il problema maggiore è trovare le modalità più congrue per superare il divario esistente tra di esse, legato alla sussistenza di funzioni, obiettivi, prospettive culturali e routine differenti.

La principale barriera è rappresentata da una eterogeneità di fondo sul piano delle teorie della conoscenza utilizzate (Rajalo and Vadi, 2017; Valimaa, 2006). A partire dagli studi pionieristici della Resnick (1987) - una delle prime studiose ad aver messo a confronto le modalità di apprendimento nei contesti formali istituzionali (scuola e università) e in quelli lavorativi e ad evidenziarne le differenze - nel corso del tempo, numerosi studiosi, tra i quali ad esempio Eraut (2004) e Hager (2004), hanno esaminato le caratteristiche della formazione universitaria e di quella che ha luogo nel contesto lavorativo (Piazza e Rizzari, 2015). Pur muovendosi da differenti prospettive e focalizzandosi maggiormente su un aspetto piuttosto che un altro, tali Autori hanno tutti sottolineato la dimensione teorica, individuale e decontestualizzata dell'apprendimento universitario, rispetto a quella pratica, condivisa e situata dell'apprendimento nel contesto lavorativo.

Si deve in particolare a Gibbons et al. (1994), la distinzione tra le due modalità di produzione della conoscenza proprie del contesto universitario e di 
quello lavorativo: mode 1 e mode 2. La prima risulta essere strettamente connessa a un ambito disciplinare specifico, nonché basata su una struttura gerarchica e immutabile nel tempo; la seconda è transdisciplinare, basata sulla collaborazione e focalizzata su problemi concreti. Stando alla teorizzazione di Gibbons, il passaggio delle università dalla mode 1 alla mode 2 è il presupposto perché si realizzi una più proficua interazione con il mondo delle imprese.

Studi successivi, come quelli di Billet (2004), di Rhoades e Slaugher (2006) e di Tynjälä (2008), hanno spostato invece l'attenzione dalla necessità di un mero adattamento del contesto universitario a quello lavorativo alla possibile creazione di nuovi modelli formativi integrati tra università e imprese. Tali studi hanno evidenziato la presenza anche nel contesto lavorativo di un apprendimento di tipo formale-strutturato, che si affianca a quelli prevalenti di natura non formale e informale, e il ruolo attivo svolto dai singoli soggetti nella determinazione della direzione degli apprendimenti stessi, in relazione ai contesti di riferimento.

A spingere verso una maggiore integrazione tra l'apprendimento universitario e quello nel contesto lavorativo è anche la constatazione del fatto che entrambi presentano dei punti di forza e dei punti di debolezza, come viene opportunamente rilevato da Tynjälä (2008). Secondo l'Autrice la promozione di abilità generali, facilmente trasferibili in diversi contesti e situazioni, finalità propria dell'istruzione formale, pur rappresentando un elemento di indubbio valore, non si rivela sufficiente a soddisfare le esigenze del contesto sociale e lavorativo attuale. D'altronde, l'apprendimento nell'ambito di un contesto specifico, risultando difficilmente trasferibile, può rivelarsi altrettanto limitante. Per questa ragione, una possibile soluzione è far sì che l'apprendimento universitario adotti alcuni caratteri di quello lavorativo e, in modo analogo, l'apprendimento nel contesto lavorativo si realizzi sfruttando i punti di forza dell'apprendimento formale universitario: l'intenzionalità, il supporto all'apprendimento e l'orientamento, l'esplicitazione della conoscenza, la concettualizzazione, il ricorso ad approcci basati sulla riflessività, il pensiero critico, la capacità di problem solving, l'apertura mentale e la resilienza (Piazza e Rizzari, 2015).

La parzialità e i limiti dell'apprendimento che ha luogo nei contesti universitario e lavorativo vengono messi in luce anche da Meredith e Burkle (2008). Secondo le due studiose, le università concentrano gran parte delle loro attività sulla concettualizzazione astratta e sulla riflessione e seguono sovente un modello formativo di tipo trasmissivo, strutturando le attività sulla base di lezioni frontali in aula senza un aggancio significativo al mondo reale. Le imprese, a loro volta, pressate dai vincoli della produzione, si concentrano soprattutto sulla dimensione pratica dell'esperienza, dedicando poco tempo alla riflessione e alla 
concettualizzazione, necessarie per promuovere il pieno apprendimento dei discenti e l'innovazione nel contesto lavorativo. La realizzazione di un apprendimento autentico e, di conseguenza, la costruzione di "ponti" tra le università e le imprese, può avere luogo, invece, soltanto mediante l'integrazione delle quattro fasi costitutive del ciclo di apprendimento di Kolb (1984): l'esperienza concreta, l'osservazione riflessiva, la concettualizzazione astratta e la sperimentazione attiva.

Una sintesi esaustiva di quanto emerso dagli studi fin qui esaminati è espressa dal modello della pedagogia integrativa (integrative pedagogy, IP), volto a supportare in modo integrato i processi di apprendimento che si realizzano nel contesto universitario e in quello lavorativo, coniugando i presupposti dell'insegnamento e dell'apprendimento costruttivista e quelli della ricerca sull'expertise professionale (Tynjälä, 2008; Tynjälä 2017; Tynjälä and Gijbels, 2012; Tynjälä et al., 2016; Virtanen and Tynjälä, 2019). In base a quest'ultima le quattro componenti fondamentali dell'expertise sono: la conoscenza fattuale, concettuale e teorica; la conoscenza pratica ed esperienziale; la conoscenza socioculturale (incorporata nelle pratiche sociali) e la conoscenza regolativa. Tradizionalmente la prima è reputata una prerogativa della formazione universitaria, mentre la conoscenza esperienziale e quella socioculturale sono considerate peculiarità del contesto lavorativo. La conoscenza regolativa, infine, intesa come capacità di metacognizione, riflessività e autoregolazione da parte del soggetto, funge da elemento di mediazione tra la formazione universitaria e quella nel contesto lavorativo. Applicando il modello della pedagogia integrativa, viceversa, lo sviluppo dell'expertise professionale e delle competenze trasversali, considerate necessarie per l'occupabilità al pari delle competenze professionali specifiche - passa attraverso l'integrazione di tutte e quattro queste componenti. Esso presuppone, inoltre, l'adozione di pratiche di apprendimento di tipo collaborativo e interattivo e l'adozione di congrui strumenti di mediazione (compiti analitici, journal e portfolio, discussioni, tutoring, mentoring, coaching) e processi di mediazione (problem solving, integrative thinking).

È evidente, dunque, che l'expertise professionale non è la semplice applicazione pratica della conoscenza acquisita, ma comporta una rielaborazione e un adattamento personali della teoria al contesto nel quale essa verrà utilizzata (Zeitler, 2018). Si tratta di concettualizzare le proprie esperienze lavorative riflettendo su di esse alla luce delle conoscenze teoriche possedute e, al contempo, di considerare le teorie in relazione al lavoro pratico svolto, operando un processo di fusione di teoria ed esperienza (Tynjälä, Valimaa and Sarja, 2003).

Questo è quanto cerca di fare il work-based learning (WBL), di cui l'apprendistato costituisce un'applicazione, attualmente reputato il modello formativo più idoneo alla coniugazione di teoria e pratica, apprendimento universitario e apprendimento nel luogo di lavoro. 
Nato nel contesto anglosassone tra la fine degli anni ' 80 e i primi anni ' 90 del secolo scorso ${ }^{3}$, ma ampiamente accreditato in ambito europeo e internazionale ormai da diversi anni, tale modello fa riferimento all'apprendimento che si realizza nel posto di lavoro, mediante lo svolgimento delle normali attività lavorative e la riflessione sulle problematiche ad esse correlate (Helyer, 2016). In senso più specifico, riferito al settore dell'istruzione superiore, il WBL fa riferimento a programmi di studio accreditati, per i quali cioè è previsto il rilascio di un titolo di studio o di una qualifica riconosciuti, svolti in collaborazione tra le università e le imprese, nei quali le problematiche del lavoro costituiscono il focus principale del curriculum, nel rispetto delle finalità educative e degli standard qualitativi delle istituzioni universitarie. Il curriculum del WBL, pertanto, non ha una natura prestabilita e basata su una struttura disciplinare, ma è di tipo transdisciplinare e viene predisposto in forma individuale, mediante un learning agreement sottoscritto dai tre attori fondamentali coinvolti nello svolgimento del programma: l'individuo che apprende (il learner), l'organizzazione lavorativa e l'istituzione accademica. La buona riuscita di un programma di WBL è legata ad alcuni fattori fondamentali: il riconoscimento degli apprendimenti precedenti; il collocamento dei risultati di apprendimento realizzati all'interno dei quadri di riferimento dei livelli e degli standard esistenti; l'offerta ai discenti di un supporto continuo all'apprendimento; lo sviluppo di una riflessione critica durante tutto il programma; la produzione di una documentazione adeguata dell'apprendimento conseguito (Piazza e Rizzari, 2015).

Tra i fattori considerati, assume particolare rilevanza soprattutto il supporto all'apprendimento offerto ai discenti durante il percorso di formazione e, pertanto, il ruolo di supervisione e di facilitazione svolto dai mentori, la cui dimensione professionale costituisce uno dei principali elementi di discussione all'interno del progetto europeo ApprEnt, qui di seguito descritto.

\section{Costruire ponti tra università e lavoro: il progetto ApprEnt}

È crescente il riconoscimento del ruolo chiave che le forme di apprendimento basato sul lavoro e, in particolare, l'apprendistato, possono ricoprire nel consentire ai giovani di acquisire le competenze necessarie a facilitare il loro passaggio dal mondo dell'istruzione al mondo del lavoro. Nella loro forma attuale, gli apprendistati in genere combinano la formazione sistematica e a lungo

\footnotetext{
${ }^{3}$ I suoi fondamenti teorici vanno rintracciati in primis nel pensiero di Dewey e, successivamente, nei diversi filoni di ricerca della ricerca-azione (includendo i diversi modelli di experiential learning), del costruttivismo sociale e della situated cognition, oltreché nelle più recenti teorie del self-directed learning e del capability approach. Per una ricostruzione diacronica si rinvia a Rizzari, 2014.
} 
termine sul posto di lavoro con l'istruzione formale, per consentire agli apprendisti di acquisire la gamma di competenze necessarie a una particolare occupazione $^{4}$. Oltre a fornire una soluzione alla disoccupazione giovanile (Ryan, 1998; Van der Velden and Wolbers, 2001; Quintini and Martin, 2006; EC, 2013; O'Higgins, 2017), l'apprendistato può favorire persone in cerca di lavoro e lavoratori adulti che, a causa dei cambiamenti nel mercato del lavoro o delle esigenze lavorative, sono tenuti a riqualificarsi o ad aggiornarsi professionalmente (CEDEFOP, 2019). Poiché gli apprendistati hanno il potenziale di dotare le persone delle competenze pertinenti per affrontare le sfide del mondo del lavoro lifelong, sono inoltre considerati un elemento importante del sistema di apprendimento permanente (ILO, 2019). I vantaggi, infine, riguardano anche le imprese che, attraverso gli apprendistati, hanno l'opportunità di migliorare la produttività e la competitività.

Nel mondo un numero crescente di paesi ha posto la sua attenzione allo sviluppo o al miglioramento dei sistemi e dei programmi di apprendistato. Questi sforzi, supportati anche dalle Raccomandazioni e dagli studi di organismi internazionali (EC, 2012, 2017; Unesco, 2015; EU, 2018 ), hanno assunto molte forme. Diversi paesi, tra l'altro, hanno modificato la legislazione, effettuato revisioni, ampliato o riprogettato i programmi di apprendistato esistenti e introdotto nuovi meccanismi di finanziamento per garantirne il funzionamento efficiente, hanno riorganizzato le strutture amministrative, rivisto i risultati e i curricula di apprendimento e lanciato campagne informative ${ }^{6}$. Per quanto l'ILO abbia messo in guardia dai pericoli dell'utilizzo dell'apprendistato come modalità per "ottenere manodopera a basso costo" e i governi siano stati invitati a

\footnotetext{
${ }^{4}$ Gli studi sull'apprendistato quale pratica formativa sono numerosi e afferenti a diversi approcci, così come le definizioni che gli sono state attribuite, focalizzate ora sul contesto di apprendimento, ora sul modello. Non è qui intenzione delle Autrici analizzare nel dettaglio le caratteristiche dell'apprendistato, per le quali si rinvia al testo di Gowlland (2014), che ne traccia alcune importanti linee di sviluppo.

${ }^{5}$ L'UE è stata particolarmente attiva nella promozione dell'apprendistato. Ha istituito, nel 2017, l'Alleanza europea per l'apprendistato (EAfA, European Alliance for Apprenticeship) e nel marzo 2018 il Consiglio dell'UE ha adottato la Raccomandazione relativa a un quadro europeo per apprendistati efficaci e di qualità (2018/C 153/01), che individua alcune caratteristiche distintive per gli apprendistati di qualità.

${ }^{6}$ Come riportato dall'ILO, le riforme dell'apprendistato hanno riguardato: 1'Africa (compresi Algeria, Benin, Burkina Faso, Ghana, Kenya, Marocco, Namibia, Sudafrica, Tunisia, Uganda, Repubblica unita di Tanzania e Zambia), le Americhe (Bahamas, Brasile, Cile, Colombia, Costa Rica, Giamaica, Messico, Perù, Stati Uniti e Trinidad e Tobago), Stati arabi (Giordania), Asia e Pacifico (Australia, Bangladesh, Cambogia, India, Indonesia, Repubblica di Corea, Myanmar e Nuova Zelanda) ed Europa (la comunità francofona di Belgio, Danimarca, Francia, Germania, Irlanda, Italia, Paesi Bassi, Norvegia, Romania, Spagna, Svizzera e Regno Unito) (ILO 2019, p. 2). Per un'analisi dell'apprendistato in Europa, si rinvia alle Thematic country Reviews avviate dal 2014 dal CEDEFOP. Per un'analisi dei sistemi di finanziamento si rinvia inoltre a CEDEFOP 2016 b.
} 
regolare e a monitorare gli apprendistati (al pari dei tirocini e di altri schemi di esperienza lavorativa), «per garantire l'offerta di un'esperienza di apprendimento reale e non sostituire i lavoratori regolari» (ILO, 2012, p. 7; ma anche Comyn and Brewer, 2018), l'apprendistato sembra rispondere efficacemente alla necessità di superamento della separazione tradizionale tra percorsi formali e apprendimento sul luogo di lavoro.

Il progetto Erasmus+ApprEnt ${ }^{7}$, di cui si presentano gli esiti nel presente contributo, è scaturito dal convincimento che l'apprendistato di alta qualità può contribuire a colmare questa distanza e che i benefici per tutti gli attori implicati siano molteplici. Gli apprendistati aiutano gli studenti a ottenere una qualifica professionale riconosciuta, mentre sviluppano abilità ed esperienze pratiche in ambienti di lavoro reale, aumentando le loro possibilità di trovare lavoro adeguato alle conoscenze e competenze possedute. I contesti lavorativi rappresentano setting apprenditivi che consentono agli studenti di entrare in contatto con la realtà lavorativa, acquisendo competenze hard e soft che sarebbero difficili da conseguire in ambienti di apprendimento artificiale (Ellström, 2010). Inoltre, gli apprendisti diventano parte della comunità di pratiche del contesto lavorativo (Wenger, 2000), collocandosi all'interno di uno specifico gruppo occupazionale, di cui possono assimilare la cultura e l'identità professionale. Per le università, i programmi di apprendistato possono promuovere apprendimenti maggiormente riflessivi, rafforzare la dimensione professionale e la pertinenza dei curricula e dei metodi di insegnamento, accelerare i contatti con il mondo del lavoro e migliorare le prospettive occupazionali degli studenti (Lester and Costley, 2010; Billett, 2011). Per le imprese il vantaggio dei programmi di apprendistato risiede nella possibilità di individuare tra gli apprendisti personale qualificato, con maggiori capacità di innovazione, che conosce le regole di funzionamento delle imprese, da inserire velocemente nel circuito produttivo, ovviando alla selezione e/o alla formazione di personale e riducendo i costi relativi (Olofsson and Panican, 2019).

Ovviamente, esistono critiche all'apprendistato che riflettono tensioni e problemi che le università devono fronteggiare nel loro rapporto con le aziende,

\footnotetext{
${ }^{7}$ Il progetto ApprEnt (Refining Higher Education Apprenticeships with Enterprises in Europe) è un progetto ERASMUS+, Support for Policy Reform, KA3 VET-Business Partnership Apprenticeships/Work-based learning, durato da ottobre 2017 a ottobre 2019. Coordinato da EUCEN (eucen European university continuing education network, BE), ha visto la partecipazione di: Université de Bretagne Occidentale (FR), Danube University of Krems (AT), Tallinn University (EE), University of Aveiro (PT), University of Turku (FI), University of Catania (IT), Universidad Complutense de Madrid (ES), Chambre Commerce Industrie Brest (FR), SENAT der Wirtschaft Österreich (AT), Estonian Chamber of Commerce and Industry (EE), Associaçao Industrial de Aveiro (PT), Federation of Finnish Enterprises, Southwest region (FI), Associación de empresarios del Henares (ES), Fundació Bosch i Gimpera (ES). Cfr. https://apprent.eucen.eu/.
} 
come già discusso nel paragrafo precedente. Integrare apprendimento sul lavoro e formazione universitaria è potenzialmente limitante se le opportunità fornite dalle aziende non corrispondono alle aspirazioni degli studenti, e se lo studente è ingabbiato in percorsi guidati dal datore di lavoro o strumentali al raggiungimento di obiettivi legati alla produzione (Usher and Solomon, 1999; Zembylas, 2006). Diversi Autori criticano le influenze delle logiche di tipo aziendale sugli apprendimenti considerati legittimi nei luoghi di lavoro, mettendo in evidenza come termini quali auto-direzione e riflessione possano essere del tutto trascurati per produrre apprendimenti maggiormente volti alla risoluzione dei problemi, privi quindi di una dimensione veramente critica (Valentin, 1999; Rhodes and Garrick, 2003; Wang, 2008). Se queste analisi suggeriscono che alcuni luoghi di lavoro possono non rappresentare ambienti di apprendimento adeguati, soprattutto nella loro relazione con le università, tuttavia le evidenze dimostrano i sostanziali vantaggi che l'apprendistato può fornire per i diversi attori (Lester and Costley, 2010).

Malgrado i benefici, va tuttavia precisato che il livello di attuazione dei sistemi di apprendistato a livello di istruzione superiore in tutta Europa è molto disomogeneo e, sebbene alcuni paesi dispongano di un quadro normativo di base, altri ne sono privi o l'apprendistato non è adeguatamente regolamentato ${ }^{8}$. La ridotta implementazione e le sfide che impediscono alle università e alle imprese di istituire e rafforzare gli apprendistati sono relative a diversi aspetti: amministrativi, legati alla definizione degli accordi e alla loro gestione secondo criteri di qualità; di progettazione di attività congruenti tra il livello universitario e quello professionale; relative al mancato possesso di abilità e competenze da parte del personale accademico nella gestione degli apprendimenti work-based; di competenze non adeguate a svolgere il ruolo di mentore da parte delle aziende (Ryan, 2012). Se molte università hanno una considerevole tradizione nell'erogare formazione professionale ${ }^{9}$, iniziale e continua, e sono quindi competenti nel supportare le imprese e gli apprendisti, il livello di sviluppo

\footnotetext{
${ }^{8}$ Per quanto riguarda gli schemi di apprendistato vigenti nei paesi membri dell'UE, si rinvia al database del CEDEFOP Cedefop European database on apprenticeship schemes. Per l'Italia, oltre a CEDEFOP, 2017, si vedano, tra gli altri: Buratti, Piovesan, Tiraboschi, 2014; ISFOL, 2016.

${ }^{9}$ L'International Standard Classification of Education (ISCED) distingue i programmi terziari con una solida base accademica e teorica (ISCED 5A) dai programmi terziari che sono maggiormente focalizzati su specifiche competenze professionali (ISCED 5B) e, quindi, associati alla formazione professionale di livello terziario. Se l'ISCED consente un confronto tra i sistemi di istruzione e i loro risultati tra $\mathrm{i}$ vari paesi, va considerato l'elevato grado di diversità delle istituzioni, della durata dei programmi, degli ambiti e delle modalità di durata dello studio, dei contenuti, dei criteri di selezione e delle qualifiche rilasciate (OECD, 2012).
} 
della formazione professionale universitaria varia considerevolmente tra i diversi paesi ${ }^{10}$.

La consapevolezza della diversità dei livelli di sviluppo e di implementazione dei modelli di apprendistato in Europa è stato il punto di partenza del progetto ApprEnt, volto a promuovere l'apprendistato attraverso lo scambio e la condivisione di buone pratiche, la creazione di materiali, l'elaborazione di un percorso di formazione per tutor e mentori. Nel coinvolgere università, camere di commercio, imprese e studenti apprendisti dei paesi partner partecipanti, il progetto ha inteso sottolineare l'importanza del lavoro sinergico tra i vari attori del percorso formativo nel progettare percorsi di apprendistato di qualità. Non di meno, il confronto e la cooperazione internazionale fra paesi con differenti livelli di sviluppo della formazione professionale di livello terziario hanno consentito di condividere e adattare agli specifici contesti le esperienze di successo già messe in campo.

Per concretizzare tali obiettivi, durante i due anni di progetto sono stati realizzati:

- report nazionali di analisi del livello di implementazione dell'apprendistato nelle singole realtà. Nei report, redatti congiuntamente da università e camere di commercio, sono stati presi in considerazione le direttive politiche esistenti e i livelli di implementazione degli apprendistati a livello regionale e/o nazionale;

- un documento di analisi delle caratteristiche chiave dell'apprendistato accademico e una SWOT analisi della cooperazione università-imprese;

- raccolta e analisi di 33 casi studio di apprendistato di successo nei diversi paesi, strutturati in base ai criteri di pertinenza, trasferibilità, impatto e potenziale di standardizzazione;

- un seminario di apprendimento (learning workshop) tra pari su modelli efficaci di apprendistato già realizzati in Francia e sul modello francese di formazione dei tutor;

- organizzazione a livello nazionale di focus group con le imprese e altri soggetti interessati al fine di individuare punti di forza e di debolezza delle specifiche realtà nazionali/regionali/locali;

- elaborazione di un prototipo di corso di formazione per mentori delle imprese e tutor accademici;

${ }^{10}$ In Italia, ad esempio, il sistema di istruzione superiore non universitario si è sviluppato attraverso tre fasi: l'Istruzione e Formazione Tecnica Superiore (IFTS) istituita nel 1999, i Poli formativi nel 2004 e gli ITS (Istituti tecnici superiori nel 2008). Gli IFTS sono programmi postaccademici post-secondari di un anno che portano a un certificato di alta specializzazione tecnica (Certificato di specializzazione tecnica superiore, EQF 4); gli istituti di Istruzione Tecnica Superiore sono programmi non accademici post-secondari di due o tre anni che portano a un diploma tecnico di alto livello (Diploma di tecnico superiore, EQF 5) (CEDEFOP, 2017). 
- redazione, in inglese e nelle lingue dei paesi partner, di: 1) un modello di accordo per l'istituzione di apprendistati da adattare alle esigenze e alle caratteristiche dei diversi paesi; 2) policy paper e raccomandazioni relativi alla promozione di apprendistati per le istituzioni di istruzione superiore e le imprese; 3) un advocacy pack comprendente linee di azione rivolte ai diversi gruppi target: università/istituti di istruzione superiore, imprese, autorità pubbliche, studenti;

- organizzazione di seminari nazionali per condividere il prototipo del corso di formazione e i documenti prodotti e identificare soluzioni in grado di facilitare l'implementazione dei programmi di apprendistato offerti delle imprese.

Il risultato complessivo del progetto va rinvenuto nella definizione di un nuovo e migliorato concetto di partnership tra gli istituti di istruzione superiore partecipanti e le aziende, necessario a rafforzare collaborazioni tra tutti gli attori e a garantire l'implementazione di apprendistati di successo.

Uno degli esiti più significativi e innovativi è l'elaborazione del corso di formazione per mentori delle imprese, il cui ruolo è ritenuto centrale (Consiglio dell'UE, $\left.2018^{11}\right)$ nei processi di apprendimento degli apprendisti. I numerosi compiti che sono richiesti ai mentori - orientare lo studente al tipo di attività prevista; aiutarlo a comprendere i learning oucomes proposti; analizzare le sue aspettative; organizzare e realizzare le esperienze nell' azienda, definendone scopi, sequenze e durata; preparare lo studente a diventare un indipendent learner; mettere in atto pratiche efficaci di apprendimento work-based; aiutare a collegare le esperienze realizzate agli apprendimenti formali già acquisiti; sostenere la riflessione dello studente circa il raggiungimento degli obiettivi del programma di formazione; valutare gli esiti dell'apprendimento - richiedono una solida preparazione pedagogica che consenta di realizzare in modo competente il mentoring (Billett, 2016).

\section{II modello di formazione dei mentori}

La figura del mentore è stata ampiamente esaminata in letteratura, ma non

\footnotetext{
${ }^{11}$ L’UE individua tra i criteri per apprendistati efficaci e di qualità il supporto pedagogico, realizzato «grazie a personale interno alle imprese, con l'incarico di collaborare strettamente con gli istituti di istruzione e formazione professionale e con gli insegnanti per fornire un orientamento agli apprendisti e garantire un riscontro reciproco e regolare. Gli insegnanti, i formatori e i tutor, in particolare nelle microimprese e nelle piccole e medie imprese, dovrebbero essere assistiti nell'aggiornare le proprie abilità, conoscenze e competenze al fine di formare gli apprendisti seguendo i metodi di insegnamento e di formazione più recenti e le esigenze del mercato del lavoro» (Consiglio dell'UE, 2018).
} 
sempre il suo ruolo e le sue funzioni sono stati definiti in modo chiaro e adeguato, anche a causa dell'uso improprio della terminologia adottata. Il mentoring è stato spesso utilizzato, infatti, come termine ombrello e usato in modo interscambiabile con altri termini come coaching, supervising, tutoring (Farquharnson, 2017; Lee, 2007; Wang and Odell, 2002; Hirst, Short and Rinne, 2014), determinando un'evidente difficoltà nell'operare una distinzione tra di essi. È abbastanza comune, ad esempio, all'interno delle imprese, attribuire al mentoring una funzione pragmatica, molto simile a quella del coaching e del training: supportare il mentee nella risoluzione di problemi inerenti alle pratiche lavorative, promuovendone soprattutto il miglioramento delle performance. Mentre però la formazione on the job è un processo che si realizza in un intervallo limitato nel tempo e su un'area specifica di lavoro, essendo finalizzata all'acquisizione delle abilità per svolgere un determinato compito lavorativo, il mentoring offre agli apprendisti un supporto costante ai processi di apprendimento che si conclude solo al termine del percorso formativo (Hirst, Short and Rinne, 2014; Bass, 2017). Il suo obiettivo fondamentale è promuovere la crescita personale del soggetto, sollecitando soprattutto la sua capacità riflessiva, intesa come processo metacognitivo di analisi e orientamento del pensiero (Lee, 2007).

Nel contesto specifico dell'apprendistato di livello terziario, il mentore supporta l'apprendimento e lo sviluppo dell'expertise professionale dell'apprendista e costruisce con lui una positiva relazione di fiducia, mettendo in atto, al contempo numerose competenze e abilità tecniche legate ai processi di managing, supervising e coaching (Hirst, Short and Rinne, 2014).

Il processo di mentoring si realizza mediante la presenza di due figure fondamentali: il tutor accademico e il mentore aziendale, aventi competenze e ruoli differenti e complementari. Il supervisore accademico ha il compito di promuovere lo sviluppo delle competenze trasversali degli apprendisti e ha padronanza soprattutto nel campo delle metodologie didattiche e dei contenuti teorici. Tra le sue funzioni rientrano: offrire consigli allo studente, accertare l'adeguatezza tra il lavoro svolto da quest'ultimo e il curriculum predisposto, tenere traccia dei suoi risultati accademici. La sfera di competenza del mentore aziendale è invece quella delle pratiche lavorative. Spettano a lui il compito di facilitare l'integrazione dell'apprendista all'interno dell'impresa e quello di definirne il percorso di apprendimento in relazione allo svolgimento dei processi lavorativi, in conformità al curriculum. A entrambi i mentori è richiesto di guidare gli apprendisti durante tutto il processo di apprendimento e di valutarne i risultati di apprendimento conseguiti (Royo et al., 2019).

Una componente fondamentale della loro azione di supporto è rappresentata dalla capacità di rendere gli studenti consapevoli delle conoscenze, competenze e abilità possedute, laddove il più delle volte queste ultime vengono date per scontate (Helyer, 2016). 
Affinché svolgano i loro compiti nel migliore dei modi, ai mentori è richiesto il possesso di numerose conoscenze, competenze e abilità, in diversi campi.

È necessario, innanzitutto, che essi abbiano adeguate competenze pedagogiche, andragogiche e psicologiche (Broek et al., 2017). Essi devono conoscere le teorie fondamentali sull'apprendimento, con riferimento in particolare all'apprendimento in età adulta e all'apprendimento learner-centred e competenceoriented (Royo et al., 2019) e possedere piena consapevolezza del significato del mentoring e del ruolo che esso riveste all'interno dello specifico contesto dell'apprendistato universitario.

Il possesso di adeguate abilità sociali (quali ad esempio l'empatia e la capacità di ascolto attivo), di competenze comunicative e interpersonali, si rivela indispensabile per interagire in modo positivo ed efficace con gli studenti e con gli altri mentori. Esse consentono, infatti, di svolgere in modo adeguato interviste e colloqui funzionali alla rilevazione delle competenze pregresse e dei bisogni di apprendimento; definire in modo chiaro gli obiettivi e i risultati di apprendimento; segnalare in modo costruttivo gli errori; dare e ricevere feedback adeguati; incoraggiare e motivare (Bass, 2017; Broek et al., 2017).

Per supportare adeguatamente il processo di apprendimento degli apprendisti, ai mentori sono richieste, altresì, capacità gestionali di analisi, di progettazione e di guidance, necessarie per comprendere le esigenze e le aspettative degli studenti e individuare, insieme agli apprendisti, percorsi e strategie di apprendimento funzionali agli obiettivi prefissati all'interno del curriculum negoziato all'inizio del percorso formativo. La sfida più grande per i mentori è proprio quella di "facilitare la difficoltà", cioè di gestire l'esperienza di apprendimento dello studente, aiutandolo a venir fuori dallo stato di confusione iniziale rispetto alle proprie esigenze formative e alla scelta del proprio progetto di ricerca, orientandolo e guidandolo verso una progressiva capacità di autodirezione nell'apprendimento (Laylock and Korpe, 2016).

Competenze indispensabili che i mentori devono possedere, infine, sono quelle valutative e di monitoraggio. I mentori devono conoscere e saper utilizzare le diverse tecniche di valutazione ed essere in grado di dare feedback adeguati, sia di tipo formativo che sommativo (Broek et al., 2017).

Nella realtà dei fatti, però, non sempre i mentori sono in possesso di tali competenze o hanno piena consapevolezza del ruolo fondamentale che esse svolgono nel determinare un adeguato supporto all'apprendimento degli studenti. A ciò si aggiunga che lo specifico dominio dell'apprendistato di livello terziario presenta elevati livelli di complessità e richiede livelli di supporto maggiori rispetto alle forme tradizionali di mentoring, poiché gli studenti devono soddisfare le richieste sia delle istituzioni accademiche sia delle organizzazioni lavorative (Royo et al., 2019; Minton and Walsh, 2016). 
Non sempre, inoltre, i mentori sono in grado di costruire un rapporto di proficua collaborazione tra loro, laddove una loro azione coordinata in tutte le fasi del programma formativo - dalla definizione iniziale degli obiettivi di apprendimento, alla scelta della strategia di apprendimento, alla documentazione degli apprendimenti, fino alla riflessione finale e alla verifica degli apprendimenti è considerata indispensabile ai fini del successo del programma di apprendistato (Broek et al., 2017).

Una formazione iniziale e continua dei mentori, in entrambi i contesti, universitario e lavorativo, si rende pertanto indispensabile (Hirst, Short and Rinne, 2014; EC, 2015). Come emerge però dai risultati delle consultazioni preliminari svolte nell'ambito del progetto ApprEnt, non tutti i paesi dell'Unione europea prevedono una formazione obbligatoria e codificata per i mentori e, anche laddove quest'ultima sia presente, spesso i mentori non vi prendono parte, percependola come una perdita di tempo o un'attività non necessaria. Anche le normative nazionali non aiutano alla definizione chiara della figura professionale dei mentori, soprattutto nel caso dei mentori aziendali, per i quali i requisiti richiesti sono il più delle volte legati agli anni di esperienza lavorativa maturata e alla posizione occupata all'interno dell'impresa piuttosto che al possesso di determinate competenze. Ad affiorare è così l'idea che il mentore non rappresenti un professionista con una identità propria, ma un lavoratore che riveste l'incarico di mentore in aggiunta ai suoi regolari compiti lavorativi (Broek et al., 2017).

Da questi presupposti è scaturita, all'interno del progetto ApprEnt, la progettazione del prototipo di un corso di sviluppo professionale continuo per i mentori, che tenesse conto delle competenze necessarie per realizzare il mentoring in contesti di apprendistato. L'obiettivo fondamentale sottostante alla progettazione del corso è stato supportare lo sviluppo delle competenze professionali dei mentori, offrendo loro occasioni di riflessione condivisa e strumenti operativi per svolgere al meglio la propria attività, anche mediante la costruzione di adeguate reti di supporto.

Per la realizzazione di un percorso formativo efficace, costruito sulla base dei reali bisogni dei partecipanti, la progettazione del corso è stata preceduta dallo svolgimento di numerosi seminari e gruppi di discussione, in presenza e online, che hanno coinvolto i principali stakeholder del progetto, sulla scorta del modello della comunità di pratica (Wenger, 2000).

Il corso è stato pensato in modo tale da avere una struttura molto generale e flessibile, di tipo modulare, tale da consentirne l'adattamento nella forma e nei contenuti alle esigenze formative, nonché ai vincoli legislativi e organizzativi presenti nei diversi contesti di applicazione. Sono stati ipotizzati, nello specifico, sette moduli formativi, della durata di circa 8 ore ciascuno, - accompa- 
gnati dalla definizione di adeguati learning outcomes - che affrontano le problematiche fondamentali connesse all'esercizio della funzione di mentoring nel contesto specifico dell'apprendistato di livello terziario e alle competenze professionali dei mentori:

1. la consapevolezza delle peculiarità e dei vantaggi dell'apprendistato;

2. l'introduzione al mentoring degli studenti;

3. la comprensione delle esigenze e delle aspettative degli studenti;

4. la pianificazione delle strategie di apprendimento degli studenti;

5. il supporto al processo di apprendimento;

6. gli strumenti per la cooperazione tra i mentori;

7. la formazione continua dei mentori.

Il modulo iniziale costituisce la premessa indispensabile allo svolgimento del corso e ha un duplice obiettivo: preparare i partecipanti alla prima sessione di lavoro ed effettuare la rilevazione delle loro competenze pregresse e dei loro bisogni di formazione, mediante la costruzione di appositi profili individuali. Almeno due settimane prima dell'inizio delle attività formative, i partecipanti al corso andranno contattati e invitati a compilare una scheda informativa contenente la descrizione del ruolo lavorativo ricoperto, dell'area di competenza, delle eventuali esperienze nella supervisione e delle aspettative nei confronti del corso di formazione. Ad essi andrà altresì fornito tutto il materiale utile da consultare per avere una prima ricognizione delle tematiche che verranno affrontate all'interno del corso: riferimenti generali alle normative nazionali ed europee dei programmi di apprendistato; riferimenti interni alle politiche e ai regolamenti istituzionali e organizzativi; studi di caso; materiali e strumenti prodotti all'interno del progetto ApprEnt.

Il secondo modulo prevede la presentazione del programma e delle motivazioni del corso. Esso ha come obiettivi fondamentali: far conoscere fra loro tutti i partecipanti, condividere le loro aspettative e motivazioni e introdurre le specificità del mentoring con gli studenti dei programmi di apprendistato di livello terziario. I temi oggetto di discussione previsti sono: la definizione di apprendistato di livello terziario e l'individuazione delle caratteristiche che lo contraddistinguono dalle altre tipologie di programmi di apprendistato; il concetto di mentore; le peculiarità del mentore accademico e di quello aziendale e le loro possibili modalità di cooperazione all'interno del programma di apprendistato.

Nel terzo modulo l'oggetto di discussione è rappresentato dalla individuazione dei bisogni e delle aspettative degli studenti - in relazione alle opportunità e alle sfide offerte dalla frequenza di un programma di apprendistato di alta formazione - e del possibile supporto che i mentori possono offrire loro. Ai partecipanti è affidato il compito di realizzare la progettazione di un curriculum di apprendistato di livello terziario, opportunamente adattato a partire da quello di un corso di studio standard. 
Il quarto modulo è incentrato sulle modalità concrete attraverso le quali i mentori possono pianificare la strategia di apprendimento degli studenti nelle sue diverse fasi di: acquisizione iniziale delle informazioni sugli studenti e sui loro bisogni di apprendimento; definizione dei risultati di apprendimento attesi; scelta condivisa con lo studente della strategia di apprendimento e del progetto di ricerca da svolgere all'interno dell'impresa; discussione della strategia di apprendimento con l'impresa e impostazione dei parametri per la verifica dei risultati di apprendimento richiesti. Un'attenzione particolare è riservata soprattutto ai metodi di debriefing, cioè alle modalità attraverso le quali i mentori possono acquisire informazioni utili dagli studenti per la pianificazione delle strategie di apprendimento.

Il quinto modulo, quello forse più importante, è incentrato sui compiti e sulle azioni di supporto dei mentori nelle diverse fasi del processo di apprendimento: definizione degli obiettivi di apprendimento (learning outcomes), scelta della strategia di apprendimento, documentazione di supporto e feedback, riflessione. Il focus è posto, nello specifico, sulle dinamiche relazionali tra i mentori e gli studenti durante tutto il percorso e sugli strumenti di documentazione dell'apprendimento comunemente usati all'interno dei programmi (diari di apprendimento o Portfolio), nonché sulle attività di debriefing e di feedback.

Nel sesto modulo le tematiche oggetto di discussione sono le modalità e gli strumenti di cooperazione tra i mentori, accademico e aziendale. L'analisi è incentrata soprattutto sui campi di cooperazione tra i due mentori (definizione del curriculum e degli obiettivi di apprendimento, progettazione del percorso di apprendimento e valutazione dei progressi degli studenti) e sugli strumenti digitali che possono essere utilizzati per favorirne la comunicazione (piattaforme Moodle condivise per l'upload dei materiali; template condivisi per la valutazione e i report; piattaforme per meeting virtuali...). L'obiettivo principale del modulo è spingere i partecipanti a riflettere sull'importanza di costruire una visione condivisa dell'apprendimento tra università e imprese e una relazione tra queste ultime basata sulla fiducia.

Il settimo modulo, conclusivo del percorso, si pone come momento di riflessione sull'esperienza di apprendimento realizzata e di autoanalisi da parte dei partecipanti dei propri punti di forza e di debolezza, nonché delle risorse a disposizione e delle strategie di miglioramento delle pratiche potenzialmente attuabili. Prefigura, quindi, la possibilità che i partecipanti applichino nei loro contesti lavorativi le conoscenze e le competenze acquisite all'interno del corso e pianifichino lo svolgimento di ulteriori esperienze di formazione.

Sul piano didattico, in linea con i presupposti teorici del modello della pedagogia integrativa, la scelta è stata quella di adottare un approccio basato sul problem solving e su metodologie attive e collaborative, prevedendo lo svolgimento di discussioni guidate, lavori in piccoli gruppi, analisi di casi concreti e 
condivisione di buone pratiche. Un valore aggiunto è rappresentato dalla ipotesi di coinvolgimento dei professionisti con adeguata esperienza nel mentoring (modelli di ruolo) e degli studenti.

\section{Considerazioni conclusive}

Il progetto ApprEnt costituisce un'esperienza di indubbio valore nell'ottica di una innovazione nelle pratiche di WBL e di apprendistato di livello terziario in Europa e di una maggiore armonizzazione delle stesse tra i diversi paesi europei. La scelta di adozione di un approccio bottom-up, basato sulla consultazione e sulla partecipazione attiva di tutti gli stakeholder, si è rivelata particolarmente efficace in tal senso, poiché ha consentito la condivisione delle buone pratiche messe in atto e il rafforzamento del dialogo e della sinergia tra le istituzioni accademiche e le imprese, oltreché l'acquisizione di utili informazioni sulle reali esigenze formative degli apprendisti e delle figure professionali coinvolte nell'implementazione dei programmi di apprendistato.

Uno dei principali punti di forza del progetto è rappresentato proprio dal prototipo di corso di formazione dei mentori, soprattutto in ragione della sua flessibilità. Esso, infatti, offre informazioni e suggerimenti significativi per orientare la costruzione del percorso formativo, garantendo però, al contempo, la libertà di adattarlo al contesto di applicazione, in relazione ai vincoli legislativi e organizzativi esistenti nei diversi paesi europei.

Nel contesto italiano, in particolare, l'implementazione del modello, in forma sperimentale, potrebbe costituire un primo passo verso una maggiore valorizzazione della figura professionale del mentore nell'ambito dei programmi di apprendistato di alta formazione. Allo stato attuale, infatti, per tale figura non è prevista alcuna formazione specifica, anche se da tempo esistono dei segnali di orientamento in questa nuova direzione. Ne costituisce un esempio il Manuale per la formazione del tutor aziendale per l'apprendistato realizzato dall'Isfol già nel $2013^{12}$. Esso, infatti, presenta diverse analogie con il prototipo di corso realizzato da ApprEnt, sia sul piano dei contenuti, che fanno riferimento specifico alle competenze implicate nell'attività di mentoring, sia sul piano della modalità di erogazione, di tipo modulare (D'Arista, 2013). Una possibile integrazione dei due modelli operativi proposti, in una dimensione riflessiva, critica e propositiva, tale da consentire l'estensione delle attività di formazione anche ai tutor del contesto universitario, potrebbe essere un primo passo verso la progettazione di un percorso formale di formazione di queste figure

\footnotetext{
${ }^{12}$ Il testo è stato proposto anche in versione web book ed è consultabile al seguente indirizzo: https://www.isfol.it/pubblicazioni/highlights/manuale-del-tutor-per-lapprendistato.
} 
professionali. Tutto ciò, ricordando che il fine ultimo del WBL, nella prospettiva dell'attuale modello della pedagogia integrativa, è promuovere la facilitazione della transizione formazione-lavoro e l'occupabilità dei giovani, soprattutto in contesti e momenti di ridotte opportunità lavorative.

\section{Riferimenti bibliografici}

Alessandrini G., a cura di (2014). Apprendistato, competenze e prospettive di occupabilità. Lecce-Brescia: Pensa Multimedia.

Bass S. (2017). Mentoring for apprenticeships. Train-the-trainer for on the-job-training (OJT). Keystone Development Partnership.

Bertagna G. (2006). Pensiero manuale. La scommessa di un sistema educativo di istruzione e di formazione di pari dignità. Soveria Mannelli: Rubettino.

Bertagna G. (2011). Lavoro e formazione dei giovani. Brescia: La Scuola.

Billett S. (2004). Workplace participatory practices: Conceptualising workplaces as learning environments. Journal of Workplace Learning, 16(6): 312-324. DOI: $10.1108 / 13665620410550295$.

Billett S. (2011). Integrating experiences in workplace and university settings: A conceptual perspective. In: Billett S. and Henderson A., eds, Developing learning professionals (pp. 21-40). Dordrecht: Springer.

Billett S. (2016). Promoting learning through work-based experience: mimetic learning in action. In: Heyler R., ed., Facilitating work-based learning. A handbook for tutors. London-New York: Palgrave.

Bocca G. (1998). Pedagogia del lavoro. Itinerari. Brescia: La Scuola.

Boffo V. (2012). Lavoro e persona: il senso, la cura, le relazioni. In: Boffo V., a cura di, Lavoro e non solo. Sguardi pedagogici (pp. 19-43). Milano: Simplicissimus Book Farm.

Brennan J., King R. and Lebeau Y. (2004). The Role of universities in the trasformation of societies: An international research project. London: Centre for Higher Education Research and Information.

Broek S.D., Cino Pagliarello M., De Vreede-Van Noort R. and Vroonhof P. (2017). Teachers and trainers in work-based learning/apprenticeships. Final report.

Brunello G. and Rocco L. (2017). The Labor Market Effects of Academic and Vocational Education over the Life Cycle: Evidence Based on a British Cohort. Journal of Human Capital, 11(1): 106-166. DOI: 10.1086/690234.

Brunello G. and Wruuck P. (2019). Skill shortages and skill mismatch in Europe: A review of the literature. EIB Working Paper 2019/05.

Buratti U., Piovesan C. and Tiraboschi M. (2014). Apprendistato: quadro comparato e buone prassi. ADAPT working paper. Testo disponibile al sito $\underline{\mathrm{http}}: / / \mathrm{adapt}$.it/adaptindice-a-z/u-buratti-c-piovesan-m-tiraboschi-cura-di-apprendistato-quadro-comparato-e-buone-prassi-adapt-labour-studies-e-book-series-n-242014 (22/03/2020).

Caliendo M. and Schmidl R. (2016). Youth unemployment and active labor market policies in Europe. IZA J Labor Policy, 5(1). DOI: 10.1186/s40173-016-0057-x. 
Cappelli P. (2014). Skill Gaps, Skill Shortages and Skill Mismatches: Evidence from the US. NBER Working Paper 20382.

CEDEFOP (2015). Tackling unemployment while addressing skill mismatch: lessons from policy and practice in European Union countries. Luxembourg: Publications Office. Cedefop research paper; No 46. DOI: 10.2801/648140.

CEDEFOP (2016a). Future skill needs in Europe: critical labour force trends. Luxembourg: Publications Office. Cedefop research paper; No 59. DOI: $10.2801 / 56396$.

CEDEFOP (2016b). Governance and financing of apprenticeships. Luxembourg: Publications Office. Cedefop research paper; No 53. DOI: 10.2801/201055.

CEDEFOP (2016c). Labour market information and guidance. Luxembourg: Publications Office. Cedefop research paper; No 55. DOI: 10.2801/72440.

CEDEFOP (2017). Apprenticeship review Italy. Building education and training opportunities through apprenticeships. Luxembourg: Publications Office of the European Union.

CEDEFOP (2018). Insights into Skill Shortages and Skill Mismatch: learning from Cedefop's European skills and jobs survey. Luxembourg: Publications Office of the European Union. Cedefop reference series. No 106. Testo disponibile al sito: http://data.europa.eu/doi/10.2801/645011 (20/03/2020)

CEDEFOP (2019). Apprenticeship for adults: results of an explorative study. Luxembourg: Publications Office of the European Union. Testo disponibile al sito: https://www.cedefop.europa.eu/files/4179_en.pdf [20/03/2020]. DOI: $10.2801 / 24300$.

Comyn P. and Brewer L. (2018). Does work-based learning facilitate transitions to decent work?. Employment Policy Department Working Paper No. 242. Geneva: ILO.

Consiglio dell'Unione Europea (2018). Raccomandazione del Consiglio del 15 marzo 2018 relativa a un quadro europeo per apprendistati efficaci e di qualità (2018/C $153 / 01)$.

Costa M. (2011). Pedagogia del lavoro e contesti di innovazione. Milano: FrancoAngeli.

D'arista F., a cura di (2013). Il tutor aziendale per l'apprendistato: manuale per la formazione. Roma: Isfol.

De Koning J., Peers Y. (2007). Evaluating active labour market policies evaluations. Discussion paper. SP I 2007-112. Testo disponibile al sito: http://core.ac.uk/download/pdf/6594782.pdf (20/03/2020).

Dewey J. (1916). Democracy and Education. New York: The Free Press (trad. it.: Democrazia ed educazione. Firenze: La Nuova Italia, 1949, VI ristampa, 1979).

Dietrichson J., Bøg M., Filges T. and Jørgensen A. (2017). Academic Interventions for Elementary and Middle School Students With Low Socioeconomic Status: A Systematic Review and Meta-Analysis. Review of Educational Research, 87(2): 4382.

Ellström P. E. (2010). Practice-based innovation: A learning perspective. Journal of Workplace Learning, 22(1/2): 27-40. 
Ericsson K. A. (2006). The influence of experience and deliberate practice on the development of superior expert performance. In: Ericsson K.A, Charness N., Feltowich P. J and Hoffmann R. R., eds, The Cambridge handbook of expertise and expert performance (pp. 685-705). Cambridge: Cambridge University Press.

European Commission (2012). Rethinking Education: Investing in skills for better socio-economic outcomes Education. Commission Communication, COM, 669.

European Commission (2013). The Effectiveness and Cost-benefits of Apprenticeships: Results of the Quantitative Analysis. Brussels: European Commission.

European Commission (2015). High performance apprenticeship \& work-based learning: 20 guiding principles. Brussels: European Commission. Testo disponibile al sito: https://www.cedefop.europa.eu/hr/toolkits/vet-toolkit-tackling-early-leaving/resources/high-performance-apprenticeships-work-based (28/03/2020).

European Commission (2017). Proposal for a COUNCIL RECOMMENDATION on a European Framework for Quality and Effective Apprenticeships. Brussels, 5.10.2017 COM (2017) 563 final.

Farquharnson M. J. (2017). Supporting Work-Based Mentors; Finding Solutions to the Current Issues that Surround Mentorship in Foundation Degrees. Thesis for the Degree of Master of Philosophy, Buckinghamshire New University Coventry University.

Federighi P., Campanile G. and Grassi C. (2012). Il Modello dell'Embedded Learning nelle PMI. Pisa: ETS.

Franchi G. (1979). Sistema d'istruzione e formazione professionale. Firenze: La Nuova Italia.

Frazer H. and Marlier E. (2010). In-Work Poverty and Labour Market Segmentation in the EU: Key lessons. Synthesis Report. EU Network of Independent Experts on Social Inclusion. Brussels: European Commission.

Frey C. B. and Osborne M. A. (2017). The future of employment: How susceptible are jobs to computerisation? Technological forecasting and social change, 114: 254280.

Gibbons M., Limoges C., Nowotny H., Schwartzman S., Scott P. and Trow M. (1994). The new production of knowledge: The dynamics of science and research in contemporany societies. London: Sage Publications.

Giustinelli P., Pavoni N. and Anelli M. (2019). From School to Work: Educational Choices Matter. Annual Report 3. Università Bocconi - IGIER.

Gowlland G. (2014). Apprenticeship as a model for learning in and through professional practice. In: Billett S., Harteis C. and Gruber H., eds, International handbook of research in professional and practice-based learning. Dordrecht: Springer.

Hager P. (2004). The conceptualization and measurement of learning at work. In: Rainbird H, Fuller A. and Munro A., eds, Workplace learning in context. London: Routledge.

Helyer R., ed. (2016). Facilitating WBL. A Handbook for tutors. London: Palgrave Macmillan.

Hirst A., Short C. and Rinne, S. (2014). The role of mentoring in supporting apprenticeships, Research paper 20, unionlearn. Testo disponibile al sito: 
https://www.unionlearn.org.uk/sites/default/files/publication/TUC\%20Mentoring\%20Role $\% 20$ Supporting\%20Apprenticeships $\% 20 \% 284 \% 29$.pdf (20/03/2020). ILO (2012). The youth employment crisis: A call for action. Resolution and conclusions of the101st Session of the International Labour Conference. Geneva: ILO (2016). Non-standard employment around the world: Understanding challenges, shaping prospects. Geneva: ILO.

ILO (2017). ILO Toolkit for Quality Apprenticeships, Volume I: Guide for Policy Makers. Geneva: ILO.

ILO (2018). World Employment and Social Outlook: Trends 2018. Geneva: ILO.

ILO (2019). A framework for quality apprenticeships. Geneva: ILO.

ISFOL (2016). Verso il sistema duale: XVI Monitoraggio sull'apprendistato Roma: ISFOL. Testo disponibile al sito: http://isfoloa.isfol.it/jspui/bitstream/123456789/1349/3/Isfol_FSE217\%20volume.pdf (20/03/2020).

Johnson W. (1978). A Theory of Job Shopping. The Quarterly Journal of Economics, 92(2): 261-278. Testo disponibile al sito: www.jstor.org/stable/1884162 $(20 / 03 / 2020)$.

Kerschensteiner G. (1912, 1926 VI ed.). Il concetto della scuola di lavoro, trad. ital. 1935, con Introduzione di G. Calò. Firenze: Bemporad-Marzocco, 1967.

Kolb D. A. (1984). Experiential learning: Experience as the source of learning and development. Englewood Cliffs, NJ: Prentice Hall.

Lave J. and Wenger E. (2006). L'apprendimento situato. Dall'osservazione alla partecipazione attiva nei contesti sociali. Milano: Erickson.

Laylock M. and Karpel M. (2016). Negotiation and work-based learning. In Helyer R., ed., Facilitating WBL. A Handbook for tutors. London: Palgrave Macmillan.

Lee A. (2007). How Can a Mentor Support Experiential Learning? Clinical Child Psychology and Psychiatry, 12(3): 333-40. DOI: 10.1177/1359104507078455.

Lester S. and Costley C. (2010). Work-based learning at higher education level: value, practice and critique. Studies in Higher Education, 35(5): 561-575. DOI: 10.1080/03075070903216635.

Levels M., van der Velden R. and Di Stasio, V. (2014). From school to fitting work: how education-to-job matching of European school leavers is related to educational system characteristics. Acta Sociologica, 57: 341-61.

Meredith S. and Burkle M. (2008). Building bridges between university and industry: theory and practice. Education \& Training, 50(3): 199-215. DOI $10.1108 / 00400910810873982$.

Minton A. and Walsh, A. (2016). Supporting work-based learners. In: Helyer R., ed., Facilitating WBL. A Handbook for tutors. London: Palgrave Macmillan.

Moon J. A. (2013). Reflection in learning and professional development: Theory and practice. London: Routledge.

Moscato M.T. (2016). Premesse per una pedagogia del lavoro: l'apprendistato come forma strutturale. Formazione, lavoro, persona, VI(16): 52-73.

O'Higgins N. (2017). Rising to the youth employment challenge: New evidence on key policy issues. Geneva: ILO. 
O'Higgins N. and Pinedo, L. (2018). Interns and outcomes: Just how effective are internships as a bridge to stable employment? Employment Working Paper No. 241. Geneva: ILO.

OECD (2012). Post-Secondary Vocational Education and Training: Pathways and Partnerships, Higher Education in Regional and City Development. Paris: OECD. DOI: 10.1787/9789264097551-en.

OECD (2016). Getting Skills Right: Assessing and Anticipating Changing Skill Needs. Paris: OECD. DOI: 10.1787/9789264252073-3-en.

OECD (2017a). Education Opportunity for All: Overcoming Inequality throughout the life course. Paris: OECD.

OECD (2017b). Getting Skills Right: Skills for Jobs Indicators. Introduction to the OECD Skills for Jobs indicators. Paris: OECD. DOI: 10.1787/9789264277878-3en.

OECD (2017c). Getting Skills Right: Good Practice in Adapting to Changing Skill Needs: A Perspective on France, Italy, Spain, South Africa and the United Kingdom Paris: OECD. DOI: 10.1787/9789264277892-en.

OECD (2019). Adult learning in Italy. What role for training funds. Paris: OECD. DOI: 10.1787/9789264311978-en.

Olofsson J. and Panican A. (2019). Labour market regulations, changes in working life and the importance of apprenticeship training: A long-term and comparative view on youth transition from school to work. Policy futures in education, 17(8): 945965.

Psacharopoulos G. and Patrinos H. A. (2018). Returns to investment in education: a decennial review of the global literature. Education Economics, 26(5): 445-458. DOI: 10.1080/09645292.2018.1484426.

Pestalozzi J.H. (1801). Abbozzo di memoriale sul legame fra istruzione professionale ed educazione. In: Becchi E., a cura di, J.H. Pestalozzi, Popolo, lavoro, educazione (pp. 174-184). Firenze: La Nuova Italia, 1979.

Piazza R. (2019). Apprendere e disapprendere nelle Smart city: l'impatto e le sfide delle nuove tecnologie digitali sull'apprendimento degli adulti. Pedagogia oggi, XVII(2): $1-14$.

Piazza R. and Rizzari S. (2015). Il modello del work-based learning. Roma: Aracne.

Purcel K., Elias P., Atfield G., Behle H., Ellison R. and Luchinskaya D. (2013). Transitions into Employment. Further Study and Other Outcomes: The Futuretrack Stage 4 Report. Warwick: IER.

Quintini G. and Martin S. (2006). Starting Well or Losing their Way? The Position of Youth in the Labour Market in OECD Countries. OECD Social, Employment and Migration Working Papers, No. 39. Paris: OECD Publishing.

Rajalo S. and Vadi M. (2017). University-industry innovation collaboration: Reconceptualization. Technovation, 62(63): 42-54. DOI: 10.1016/j.technovation.2017.04.003.

Resnick L.B. (1987). Learning in school and out. Educational Researcher, 16: 13-20. DOI: $10.2307 / 1175725$.

Rhoades G. and Slaughter S. (2006). Mode 3, Academic capitalism and the new economy: Making Higher Education work for whom? In: Tynjälä P., Välimaa J. and 
Boulton-Lewis J, eds, Higher Education and Working Life: Collaborations, Confrontations and Challenges. The Netherlands: Elsevier Ltd.

Rhodes C. and Garrick J. (2003). Project-based learning and the limits of corporate knowledge. Journal of Management Education, 27(4): 447-71.

Rizzari S. (2014). Tra università e lavoro: il modello formativo del work-based learning. I problemi della pedagogia, 2: 341-369.

Royo C., Uras F., Krüger K., Grundschober I. and Frasch R., eds. (2019). Training for Mentors and Supervisors of Higher Education ApprEnticeship programmes - A Generic Structure for a Continuing Professional Development Course. ApprEnt tools for Higher Education Apprenticeship in Europe series.

Ryan P. (1998). Is apprenticeship better? A review of the economic evidence. Journal of Vocational Education \& Training, 50(2): 289-325.

Ryan P. (2012). Apprenticeship: Between theory and practice, school and workplace. In: Pilz M., ed., The Future of Vocational Education and Training in a Changing World. Wiesbaden: VS Verlag für Sozialwissenschaften, pp. 402-432.

Schön D. (1983). The reflective practitioner: How professionals think in action. New York: Basic Books.

Schön D. A. (1993). Il Professionista riflessivo: per una nuova epistemologia della practica professionale (Vol. 152). Roma: Edizioni Dedalo.

Sutherland Olsen D. and Tikkanen T. (2018). The developing field of workplace learning and the contribution of PIAAC. International Journal of Lifelong Education, 37(5): 546-559. DOI: 10.1080/02601370.2018.1497720.

Tomassini M. (2016). Overcoming the low-learning scar effect: Narratives of learning and resilience of Italian low-skilled. British Journal of Guidance and Counselling, 44: 185-197.

Topel R.H. and Ward M.P. (1992). Job mobility and the careers of young men. The Quarterly Journal of Economics, 107(2): 439-479.

Tynjälä P., Virtanen A., Klemola U., Kostiainen E. and Rasku-Puttonen H. (2016). Developing social competence and other generic skills in teacher education: applying the model of integrative pedagogy. European Journal of Teacher Education, 39(3): 368-387. DOI: 10.1080/02619768.2016.1171314.

Tynjälä P. (2008). Perspectives into learning at the workplace. Educational Research Review, 3: 130-154. DOI: 10.1016/j.edurev.2007.12.001.

Tynjälä P. (2017). Pedagogical Perspectives in Higher Education Research. In: Encyclopedia of International Higher Education Systems and Institutions (pp. 1-6). Springer Netherlands. DOI:10.1007/978-94-017-9553-1_170-1.

Tynjälä P. and Gijbels D. (2012). Changing world: Changing pedagogy. In: Tynjälä P., Stenström M.L. and Saarnivaara M., eds., Transitions and Transformations in Learning and Education. Dordrecht: Springer.

Tynjälä P., Välimaa J. and Sarja A. (2003). Pedagogical perspectives on the relationships between Higher Education and working life. Higher Education, 46(2): 147166. DOI: $10.1023 / \mathrm{A}: 1024761820500$.

UNESCO (2015). Recommendation concerning technical and vocational education and training (TVET). Testo disponibile al sito: http://portal.unesco.org/en/ev.php- 
$\underline{\mathrm{URL} I D=49355 \& U R L \_D O=D O \quad T O P I C \& U R L \_S E C T I O N=201 . \mathrm{html}}$ $(28 / 03 / 2020)$.

Usher R. and Solomon N. (1999). Experiential learning and the shaping of subjectivity in the workplace. Studies in the Education of Adults, 31(2): 155-63.

Valentin C. (1999). Challenging the corporation: Critical perspectives on workplace learning. Paper presented at Standing Conference for University Teaching and Research in the Education of Adults, 29th Annual Conference, July 5-7, University of Warwick.

Välimaa J. (2006). Analysing the relationship between Higher Education institutions and working life in a nordic context. In: Tynjälä P., Välimaa J. and Boulton-Lewis J., eds., Higher Education and Working Life: Collaborations, Confrontations and Challenges. The Netherlands: Elsevier Ltd.

Van der Velden R. and Wolbers W.M. (2001). The Integration of Young People into the Labour Market within the European Union: The Role of Institutional Settings. Research Centre for Education and the Labour Market. Working paper No. 2001/7E. Maastricht: Maastricht University.

Verhaest D. and Omey E. (2013). The relationship between formal education and skill acquisition in young workers' first jobs. Manchester School, 81: 638-59.

Verhaest D., Lavrijsen J., Van Trier W., Nicaise I. and Omey E. (2018). General education, vocational education and skill mismatches: short-run versus long-run effects. Oxford Economic Papers, 70(4): 974-993.

Virtanen A. and Tynjälä P. (2019). Factors explaining the learning of generic skills: a study of university students' experiences. Teaching in Higher Education, 24(7): 880-894. DOI: $10.1080 / 13562517.2018 .1515195$.

Wang J. and Odell S. J. (2002). Mentored learning to teach according to standardsbased reform: A critical review. Review of Educational Research, 72(3), 481-546. DOI: $10.3102 / 00346543072003481$.

Wang L. (2008). Work-based learning: A critique. International Journal of Learning 15(4): $189-96$.

Wareing M. (2008). Foundation degree students as work based learners: the mentor's role. British Journal of Nursing, 17(8), 532-37.

WEF. World Economic Forum (2019). Strategies for the new economy: Skills as the currency of the labour market. Testo disponibile al sito: https://www.weforum.org/whitepapers/strategies-for-the-new-economy-skills-as-the-currency-ofthe-labour-market $(22 / 03 / 2020)$.

Wenger E. (2000). Communities of practice and social learning systems. Organization $7(2): 225-246$.

Wieland C. (2018). Apprenticeship training in Italy - a cost effective model for firms? Guetersloh: Bertelsmann Foundation.

Zeitler A. (2018), Apprenticeship skills development and 'vocationalisation': pedagogical and didactics perspectives. In: ApprEnt. Refining HE Apprenticeships with Enterprises in Europe, Learning Workshop, Brest (FR), 15 maggio 2018. 